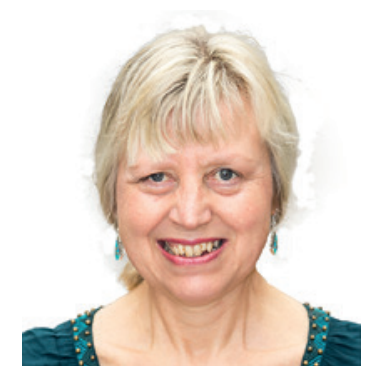

\title{
Food Insecurity and Malnutrition
}

\author{
Amanda Avery
}

Division of Food, Nutrition \& Dietetics, University of Nottingham, Sutton Bonington Campus, Leicestershire, UK

Abstract from Christian VJ, MillerKR, Martindale RG: Food Insecurity, Malnutrition, and the Microbiome. Curr Nutr Rep. 2020;DOI: 10.1007/ s13668-020-00342-0.

\section{Keywords}

Food insecurity - Food desert - Hunger - Malnutrition .

Microbiome $\cdot$ Microbial immaturity · Dysbiosis, Probiotic

\begin{abstract}
Purpose of Review: Food insecurity is defined as the disruption of food intake or eating patterns because of lack of money and other resources. Although a relationship between food insecurity and malnutrition would seem intuitive, this relationship remains unclear. This review was aimed at summarizing the emerging evidence of the relationship between food insecurity, malnutrition, and intestinal microbial changes.
\end{abstract}

Recent Findings: Evidence suggests that malnutrition is certainly associated with alteration in the intestinal microbiome. Alternative theories have been proposed as to the nature of the alteration, whether that be a result of microbial immaturity, dysbiosis, or contributions from both processes.

Summary: Although the evidence continues to evolve, targeted nutritional therapies in conjunction with specific microbial therapy of probiotics or prebiotics may hold great promise for the treatment of malnutrition in the clinical and community settings. ๑ 2020 Springer Science+Business Media, LLC, part of Springer Nature 


\section{Knowledge Transfer}

\section{Background}

'without nutritious food, there is sub-optimal health'

Food insecurity occurs when people are regularly worried or concerned about their ability to access adequate amounts of safe, affordable, and nutritious foods [1]. It is a global problem and can occur because of a lack of income to afford healthier foods or because healthier food choices are not easily available. The latter can occur for a number of reasons. For example, anything that affects the food supply chain may affect access to healthier foods. Or a person's circumstances may mean that it is harder for them to access healthier foods, and these circumstances can be multifactorial - perhaps because they have limited transport or limited storage/ cooking facilities. Some people are able to adapt to circumstances to maintain a reasonably nutritious diet, whilst others are more susceptible to relative changes in their levels of food insecurity [2]. Food insecurity, whether a relative change or absolute severity, may increase the risk of malnutrition in all of its forms [1, 3]. Certain groups of the population are more vulnerable to food insecurity and thus more susceptible to dietary inadequacies. Of concern is that these vulnerable groups include pregnant mothers, families with children, the elderly, and people on a low income or dependent on state benefits who already experience wider health inequalities.

\section{Effects of the COVID-19 Pandemic on Food Insecurity}

The COVID-19 pandemic has led to economic and social crises, and the prevalence of global food insecurity has increased [4]. We have become more aware of food insecurity, whilst in the past it has been a hidden cause of malnutrition. Unemployment, under-employment, and employment uncertainty are the reality for many people due to the pandemic. COVID-19-related global lockdowns affected all stages of the food supply chain from farm to fork, including processing and production, distribution, and consumption [5]. The United Nations World Food Program estimated that 265 million people, a two-fold increase, faced acute food insecurity by the end of 2020 [6]. Two USA surveys have found that the COVID-19 pandemic has led to far higher rates of food insecurity in households with children than in previous years [7]. The first reported that 34.4\% of households with children $\leq 12$ years old were food insecure by the end of April 2020, compared with 15.1\% in 2018. The Survey of Mothers with Young Children reported similar household food insecurity among $40.9 \%$ of mothers with children $\leq 12$ years old, with $17.4 \%$ claiming that "the children in my household were not eating enough because we just couldn't afford enough food", compared with 3.1\% in 2018. The elderly living alone, managing on limited incomes, have become more isolated and socially detached during the lockdown periods and have struggled more to access their normal support. The causes of food insecurity and under-nutrition amongst older people are multiple, and increased isolation may have further negatively impacted general physical and mental health, appetite, food availability and supply, as well as family, social and care support [8].

\section{Forms and Consequences of Food Insecurity}

Food insecurity does not just affect the amount of food which is eaten but also influences food choice. Food insecurity is generally considered to be linked to the preference of processed foods over foods of high nutritional value such as vegetables and fruits due to a 'substitution' effect where higher-quality and/or less calorie-dense foods are replaced with more perceived filling energy-dense foods, often high in simple carbohydrates and fat, that are cheaper on a per-calorie basis [9]. Cross-sectional studies support this hypothesis in both children and adults. In a study of 3,605 children aged 9-11, Fram et al. found that child food insecurity was associated with a lower Healthy Eating Index (HEI) vegetable subscale score and a higher intake of energy, fat, and sugar [10]. Child food insecurity was not associated with fruit intake or total HEl score in this study, with other studies suggesting that reduced fruit may be compensated by an increased fruit juice intake [11]. In a sample of just over 2,000 children aged 13-17 in Ethiopia, Belachew et al. reported that food insecurity was associated with a lower intake of animal-based food, protein-rich food, dairy products, and fruit. Food insecurity was also associated with lower diet diversity and food variety scores [12]. Food insecurity was found to be associated with a lower total $\mathrm{HEl}$ score and a higher intake of added sugars and empty calories in adults participating in the 2003-2010 National Health and Nutrition Examination Surveys (NHANES) [13]. In a comprehensive review of the literature, food insecure adults consumed fewer fruit, vegetables, and dairy products [14]. The 2016 National Nutrition Screening Survey suggests that around $1.3 \mathrm{~m}$ people aged 65 years and older in the UK do not get sufficient energy and protein in their diets [15]

Maternal food insecurity during pregnancy may cause lower-thanrecommended weight gain and is associated with intrauterine growth restriction [1]. For children and adolescents, exposure to food insecurity may be associated with impaired growth and physical development, cognitive deficits and reduced educational attainment, chronic physical and mental health problems, and increased mortality [16]. Food insecurity has cognitive and behavioural implications for parents and caregivers, including skipping meals due to limited income, hoarding food, and pressuring or overfeeding children when food is available because of worry about running out of food. It can negatively impact overall child well-being through the associated family conflict. Stress, bouts of starvation, secretive eating, and food cravings during times of financial hardship may promote binge-eating episodes in both children and adults [17]. Food insecurity is positively associated with levels of obesity in females perhaps due to a combination of changes in food choice and changes in eating behaviours [18]. In contrast, the associated under-nutrition is a major cause of functional decline and mortality amongst older people. It can lead to poorer health 
outcomes, falls and fractures, delays in recovery from illness, and longer periods in hospital [8].

Of increasing interest is the impact that food insecurity can have on the gut microbiota, and it has been reported that gut microbiota composition and function are altered in nutritionally depleted states [19]. A healthy gut microbiome is dependent on a healthy and diverse diet for all ages. We know that a diversity in fibre intake from minimally processed foods is beneficial. In contrast, a diet high in refined carbohydrate and low in vegetables and whole fruits may lead to dysbiosis [20].

\section{Conclusion}

What needs to be done to address the malnutrition associated with food insecurity? There is probably not one single solution, and therefore cost-effective multisectoral interventions are needed. For example, subsidising healthier foods, taxing foods and drinks providing empty calories, providing incentives for the production of traditional nutrition-rich staples, influencing healthier food choice through accessible nutrition labelling and nutrition education, and providing subsidised healthy meals at nurseries, schools, in the workplace, and at luncheon clubs might be some potential solutions. The importance of a nutrition-sensitive supply chain should also be recognised. It is important to identify barriers at any point in the supply chain so healthier foods can be distributed ethically and appropriately. Clearly, a sustainable and inclusive economic growth, which aims to reduce income, educational, age, and gender inequality, should be a key policy goal in the successful fight against food insecurity and malnutrition.

\section{Disclosure Statement}

I hereby declare that there are no conflicts of interest with regard to this commentary.

\section{References}

1 Maitra, C. 2018. A review of studies examining the link between food insecurity and malnutrition. Technical Paper. FAO, Rome. 70 pp. Licence: CC BY-NC-SA 3.0 IGO. (Available at http://www.fao.org/3/CA1447EN/ca1447en. pdf)

2 Armstrong B, Hepworth AD, Black MM. 2020. Hunger in the household: Food insecurity and associations with maternal eating and toddler feeding Pediatr Obes. 15(10):e12637. doi: 10.1111/ijpo.12637. Epub 2020 Apr 15. PMID: 32294800.

3 Thompson B, Cohen MJ, Meerman J. 2012. World Food Insecurity and Malnutrition: Scope, Trends, Causes and Consequences. In: Thompson B, Cohen M. (eds) The Impact of Climate Change and Bioenergy on Nutrition. Springer, Dordrecht. https://doi.org/10.1007/978-94-007-0110-6_3
4 Paslakis G, Dimitropoulos G, Katzman DK. 2021. A call to action to address COVID-19-induced global food insecurity to prevent hunger, malnutrition, and eating pathology. Nutr Rev. 79(1):114-116. doi:10.1093/nutrit/nuaa069

5 Torero M. 2020. Without food, there can be no exit from the pandemic. Nature. 580(7805):588-589. doi: 10.1038/d41586-020-01181-3. PMID: 32327745 .

6 UN World Food Programme 'Risk of hunger pandemic as coronavirus set to almost double acute hunger by end of 2020' www.wfp.org (accessed 12.03.21)

7 Bauer L. 2020. The COVID-19 Crisis Has Already Left Too Many Children Hungry in America. (accessed on 12.03.21); Available online: https://www. brookings.edu/blog/up-front/2020/05/06/the-covid-19-crisis-has-alreadyleft-too-many-children-hungry-in-america/

8 Purdam K, Esmail A, Garratt E. (2019) Food insecurity amongst older people in the UK. Br Food Journal. 121 (3), pp. 658-674 doi:10.1108/BFJ-05-20180301

9 Morales ME, Berkowitz SA. (2016) The Relationship between Food Insecurity, Dietary Patterns, and Obesity. Curr Nutr Rep. 5(1):54-60. doi:10.1007/ s13668-016-0153-y

10 Fram MS, Ritchie LD, Rosen N, Frongillo EA. (2015). Child experience of food insecurity is associated with child diet and physical activity. The Journal of nutrition. 145:499-504

11 Lee J, Kubik MY, Fulkerson JA. (2019). Diet Quality and Fruit, Vegetable, and Sugar-Sweetened Beverage Consumption by Household Food Insecurity among 8- to 12-Year-Old Children during Summer Months. J Acad Nutr Diet. 119(10):1695-1702. doi:10.1016/j.jand.2019.03.004

12 Belachew T, Lindstrom D, Gebremariam A, et al. (2013). Food insecurity, food based coping strategies and suboptimal dietary practices of adolescents in Jimma zone Southwest Ethiopia. PloS one. 8:e57643.

13 Nguyen BT, Shuval K, Bertmann F, Yaroch AL. (2015). The Supplemental Nutrition Assistance Program, Food Insecurity, Dietary Quality, and Obesity Among U.S. Adults. American Journal of Public Health. 105:1453-1459.

14 Hanson KL, Connor LM. (2014). Food insecurity and dietary quality in US adults and children: a systematic review. Am J Clin Nutr. 100(2):684-92. https://doi.org/10.3945/ajcn.114.084525.

15 BAPEN (2016), 'Annual report', British Association of Parenteral and Enteral Nutrition, Redditch.

16 Jones AD. (2017). Food insecurity and mental health status: a global analysis of 149 countries. Am J Prev Med. 53:264-273.

17 Armstrong B, Hepworth AD, Black MM. (2020). Hunger in the household: food insecurity and associations with maternal eating and toddler feeding. Pediatr Obes. doi: 10.1111/ijpo.12637.

18 Nettle D, Andrews C, Bateson M. (2017). Food insecurity as a driver of obesity in humans: The insurance hypothesis. Behav Brain Sci. 40:e105. doi: 10.1017/S0140525X16000947

19 Christian VJ, Miller RJ, Martindale RG. (2020). Food Insecurity, Malnutrition, and the Microbiome. Current Nutrition Reports https://doi.org/10.1007/ s13668-020-00342-0

20 De Filippis F, Pellegrini N, Vannini L, et al. (2016). High-level adherence to a Mediterranean diet beneficially impacts the gut microbiota and associated metabolome. Gut. 65(11):1812-1821. doi: 10.1136/gutjnl-2015-309957. Epub 2015 Sep 28. PMID: 26416813.

Correspondence to:

Dr. Amanda Avery, amanda.avery@nottingham.ac.uk 